Chinese Journal of Organic Chemistry

\title{
石墨烯共价修饰中的有机反应
}

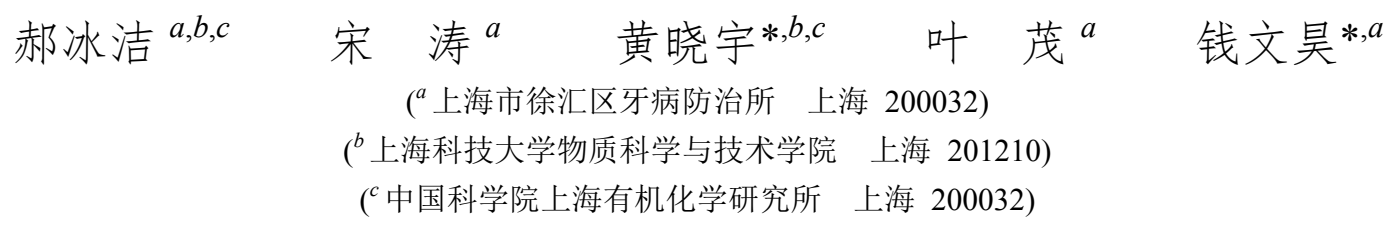

\begin{abstract}
摘要 石墨烯及氧化石墨烯结构独特, 理化性质优异, 近年来成为生物、信息、能源等领域极具应用潜力的材料. 基于 石墨烯类材料, 与功能分子、聚合物和纳米粒子等杂化, 可得到性能优越的复合材料. 除了基于弱相互作用的修饰外, 通过有机反应对石墨烯及氧化石墨烯实现共价修饰，可稳定有效地优化其结构、增强其性能、扩展其应用. 综述了近 年来化学共价修饰石墨烯及氧化石墨烯的酯化反应、酰化反应、Williamson 反应和 Claisen 重排反应、Click 化学等途 径, 阐述了有机化学在共价功能化石墨烯中的应用, 并展望了其未来发展趋势.
\end{abstract}

关键词 石墨烯; 氧化石墨烯; 共价修饰; 有机反应

\section{Organic Reactions in Covalent Functionalization of Graphene}

\author{
Hao, Bingjie Song, Tao $^{a} \quad$ Huang, Xiaoyu ${ }^{*, b, c} \quad$ Ye, Mao $^{a} \quad$ Qian, Wenhao ${ }^{*, a}$ \\ ( ${ }^{a}$ Department of Stomatology, Shanghai Xuhui District Dental Center, Shanghai 200032) \\ ( ${ }^{b}$ Shanghaitech University, School of Physical Science and Technology, Shanghai 201210) \\ ( ${ }^{c}$ Shanghai Institute of Organic Chemistry, University of Chinese Academy of Sciences, \\ Chinese Academy of Sciences, Shanghai 200032)
}

\begin{abstract}
Graphene and graphene oxide possess unique structure and excellent properties, and have become popular potential materials in biology, information, energy and other fields in recent years. The high-quality nanocomposites were obtained by hybridizing graphene-based materials with functional molecules, polymers and nanoparticles. Besides the modification via weak interaction, covalent modification of graphene and graphene oxide via organic reaction can stably and effectively optimize the structure, enhance their performances and extend their applications. In this review, the diverse approaches of chemically covalent modification of graphene and graphene oxide are reviewed via esterification, acylation, Williamson reaction, Eschenmoser-Claisen $[3,3] \sigma$ rearrangement and click chemistry, and the future development trend is prospected.

Keywords graphene, graphene oxide; covalent functionalization; organic reactions
\end{abstract}

人类的文明发展与材料的更新换代息息相关，新材 料的诞生以及使用为人类的生存繁衍奠定坚实基础. 作 为碳材料家族的新成员, 二维石墨烯 (graphene) 的出现 为人类社会的进步带来新的契机 ${ }^{[1-2]} .2004$ 年, Geim 和 Novoselov 等 ${ }^{[3-5]}$ 报道了通过胶带剥离高定向石墨, 并利 用显微镜找到了理论厚度仅为 $0.34 \mathrm{~nm}$ (约为头发直径的 二十万分之一)的石墨烯, 证实了石墨烯的真实存在,
并因此获得了 2010 年诺贝尔物理学奖. 理想的石墨烯 片层是由碳原子堆积形成的单层或几层的蜂窝状晶格 结构, 基本单元为六元环以及少量的五元环和七元环, 具有褶皱、大 $\pi$ 键、超共轭平面等微观结构, 奠定了其 独特的物理化学性质 ${ }^{[6]}$. 碳原子间可形成较强的 $\sigma$ 键, 使得石墨烯在二维尺度上具有优异的刚性 ${ }^{[7]}$, 而且石墨 烯能够通过弯曲等方式保持结构稳定性，稳定的晶格结

\footnotetext{
* Corresponding authors. E-mail: pingyanlaoto@163.com; xyhuang@mail.sioc.ac.cn

Received April 14, 2020; revised May 4, 2020; published online May 11, 2020.

Dedicated to Professor Henry N. C. Wong on the occasion of his 70th birthday.

Project supported by the National Natural Science Foundation of China (No. 51773222), the Shanghai Scientific and Technological Innovation Project (No. 20ZR1452200), the Scientific Research Project of Science and Technology Commission of Xuhui Municipality (No. SHXH201613), the Scientific Research Project of Xuhui Provincial Commission of Health and Family Planning (No. SHXH201706), the Program for Outstanding Medical Academic Leader (No. 2019LJ27) and the Shanghai Medical Key Specialty (No. ZK2019B12)

国家自然科学基金(No. 51773222)、上海市自然科学基金(No. 20ZR1452200)、上海市徐汇区科学技术委员会(No. SHXH201613)、上海市徐汇区医学
} 尖峰学科(No. SHXH201706)、上海市医学领军人才(No. 2019LJ27)和上海市医学重点专科(No. ZK2019B12)资助项目. 
构使得石墨烯具有超高的机械强度和柔韧性 ${ }^{[8]}$. 石墨烯 在垂直于晶面方向上, 每个碳原子可贡献一个未成键的 $\mathrm{p}$ 轨道 $\pi$ 电子, 形成垂直于晶面方向上大 $\pi$ 键, 大 $\pi$ 键中 $\pi$ 电子的自由移动, 赋予了石墨烯优异的导电性能 ${ }^{[6]}$. 石墨烯独特的化学结构, 优异的电、热、力及吸附等性 质，使得其在光电、能源、信息等领域有着潜在的广泛 应用, 成为近年来纳米领域研究的热点 ${ }^{[9-12]}$.

氧化石墨烯(graphene oxide)作为富含羟基、环氧、 酯基和羧基等含氧基团的石墨烯衍生物, 也被认为是通 过化学或热还原法合成石墨烯的前驱体 ${ }^{[13-17]}$, 因其较好 的水相分散性、聚合物兼容性以及生物相容性, 在生物、 催化、能源等领域有着极大的应用前景 ${ }^{[18-21]}$. 氧化石墨 烯具有与石墨烯类似的单片层准二层结构, 但是其结构 中富含 $\mathrm{OH} 、 \mathrm{COOH} 、 \mathrm{O}$ 和 $\mathrm{C}=\mathrm{O}$ 等含氧官能团. 目前被 广泛接受的氧化石墨烯结构为 1998 年提出的 LerfKlinowski 模型 ${ }^{[22]}$, 该模型中, 氧化石墨烯的平面碳原 子以 $\mathrm{sp}^{2}$ 相互成键, 碳层平面接有羟基及环氧基, 边缘 连接羧基、羰基、苯酚、内酯和醌等官能团. 由于含氧 基团在氧化石墨烯碳层中的不均匀分布, 造成碳层平面 中 $\mathrm{sp}^{2}$ 碳与 $\mathrm{sp}^{3}$ 碳交叉分布, 在氧化石墨烯表面形成大量 褶皱, 使得氧化石墨烯平面存在一定缺陷. 氧化石墨烯 中含氧官能团分布的不确定性造成其纳米结构上的不 均匀性, 影响了其在电子、机械和电化学等方面性质 ${ }^{[23]}$. 但是, 氧化石墨烯所富含的活性含氧基团可提高其亲水 性、聚合物兼容性及生物相容性, 并可作为氧化石墨烯 的化学修饰或功能化位点, 进而用于通过共价键或非共 价键固定各种功能分子, 进一步增强氧化石墨烯的功能 性，扩宽氧化石墨烯的应用 ${ }^{[24-30]}$. 氧化石墨烯的易合成 性、聚合物兼容性、溶液可加工性及其多功能性, 使其 在基础研究和应用中具有吸引力.

石墨烯及其衍生物氧化石墨烯因其丰富的原料、经 济的制备方式、独特的结构、优异的理化性质在生物、 信息、能源等领域有着极大的应用潜力. 利用石墨烯或 氧化石墨烯与聚合物、纳米颗粒、生物大分子等 $\pi-\pi$ 相 互作用、离子键及氢键等弱相互作用, 可将功能分子固 定在石墨烯与氧化石墨烯表面, 得到新的性能优异的复 合材料 ${ }^{[31-33]}$. 但是基于弱相互作用所构建的石墨烯类复 合材料在苛刻条件下理化性质不稳定，进而限制其应用 范围, 难以得到稳定、高效、普适的石墨烯类杂化功能 性材料. 为进一步扩展石墨烯类功能材料的应用范围, 增强石墨烯及氧化石墨烯的功能化, 近年来, 合成化学 家们利用石墨烯和氧化石墨烯独特的表面结构及活性 基团, 发展了一系列改性石墨烯和氧化石墨烯的化学方 法, 包括酯化反应、硅烷化反应、Williamson 反应和 Claisen 重排反应等 ${ }^{[34-38]}$. 全面概述了近期有机化学反
应共价修饰石墨烯及氧化石墨烯的合成研究进展，着重 介绍了酯化反应、硅烷化反应、Williamson 反应和 Claisen 重排反应、Click 化学等途径, 并对功能小分子 和聚合物共价修饰的特点及优劣进行了梳理, 以期为进 一步开展相关研究工作提供参考

\section{1 石墨烯的共价修饰}

目前, 有机功能分子共价修饰的石墨烯片层已在多 领域发挥其应用价值. 最为关键的是, 有机功能分子共 价修饰后的石墨烯可有效改善其在常用有机溶剂中的 分散性, 增强石墨烯的兼容性, 为构建石墨烯纳米复合 材料奠定坚实的基础. 此外, 特征活性官能团的引入可 使石墨烯具有新的结构特性，也可影响其原有共轭六元 环的芳香性，进而调控石墨烯的导热性、导电性等. 目 前，直接对石墨烯表面的碳原子进行共价修饰难以实 现, 因为该片层上几乎所有的碳原子都是饱和的 $\mathrm{sp}^{3}$ 结 构, 很难通过化学发应引入新的基团. 通过有机反应对 石墨烯进行共价修饰一般有两条途径: (1)自由基或亲双 烯体分子与石墨烯表面所含较少的 $\mathrm{sp}^{2}$ 碳的 $\mathrm{C}=\mathrm{C}$ 键加 成形成新的共价键, 引入新的杂原子官能团; (2)有机官 能团与石墨烯前驱体即氧化石墨烯所含的活性含氧官 能团之间反应形成新的共价键, 引入多样性的基团, 再 经过还原得到化学共价修饰的石墨烯复合物. 与目前已 广为人知的富勒烯和碳纳米管类比, 可与石墨烯表面 $\mathrm{sp}^{2}$ 碳反应的, 较为常见的反应物，包括活性有机自由基 和亲双烯体分子.

\section{1 石墨烯表面 $\mathrm{sp}^{2}$ 碳的自由基加成}

重氮盐加热可产生一种高活性的自由基, 用以攻击 石墨烯平面上的 $\mathrm{sp}^{2}$ 碳原子, 形成新的共价键, 实现对 石墨烯的共价化学修饰. 2010 年, Tour 等 ${ }^{[39]}$ 利用重氮化 反应，对石墨烯片层进行共价修饰，得到了硝基苯共价 功能化的石墨烯片层. 如图 1 所示, 利用重氮盐进行石 墨烯共价化学修饰时, 其片层中的 $\mathrm{sp}^{2}$ 碳转化为 $\mathrm{sp}^{3}$ 碳, 芳香体系被破坏, 超共轭平面减弱, 随着反应程度的加 深, 石墨烯电导率显著下降. 反应过程监测发现, 利用 重氮化方法共价修饰石墨烯, 改变其化学机构, 可有效 调控石墨烯片层的电导率，在半导体材料领域有着极大 的应用潜力. 此外, Barron 等 ${ }^{[40]}$ 报道了一种简单高效的 方法, 将未氧化的石墨粉通过超声分散于二氯苯溶剂 中, 制备得到了石墨烯片层的均匀分散液, 紧接着通过 两种不同的自由基反应，在石墨烯片层表面接枝上新的 修饰基团, 相应的电学测试结果表明, 化学共价修饰后 的石墨烯具有更加优异的导电性能. 此前也曾报道, 通 过重氮化以及酯化反应制备得到石墨烯片层表面, 引入 含溴的引发基团, 接着通过原位单电子转移活性自由基 

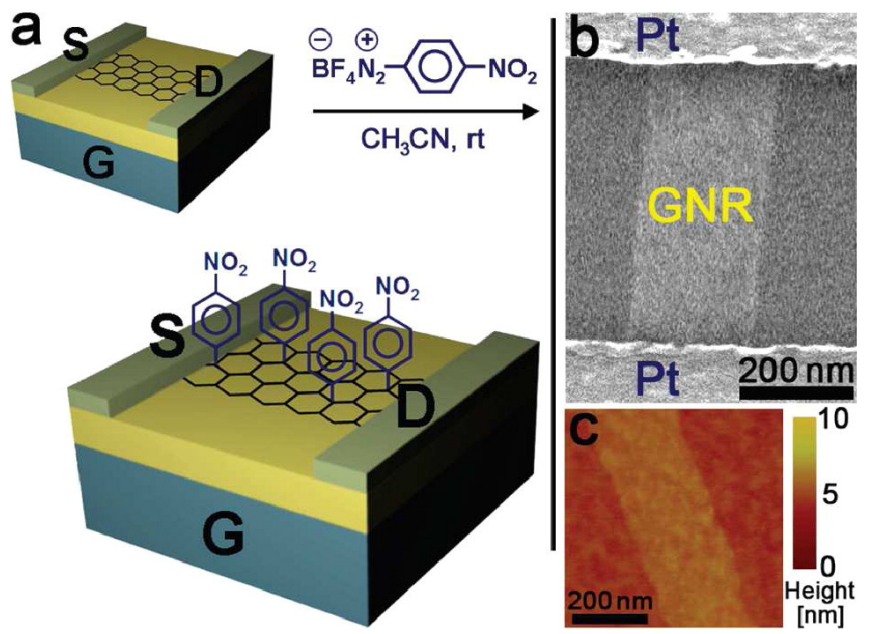

图 1 (a)4-硝基苯修饰石墨烯示意图、(b)石墨烯覆盖 Pt 电极 的 SEM 图像和(c)单层石墨烯片层的 AFM 图像 ${ }^{[39]}$

Figure 1 (a) Schematic representation of chemical doping of graphene with 4-nitrophenyl groups, (b) SEM image of a graphene nanoplatelet between Pt electrodes, and (c) AFM image of a fragment of a monolayer graphene ${ }^{[39]}$

聚合(SET-LRP), 在较为温和的条件下制备得到了聚合 物分子刷共价功能化的石墨烯片层, 通过自由基反应在 石墨烯片层表面接枝温敏性聚合物, 该聚合物共价修饰 的石墨烯复合材料可表现出较好的温敏性 ${ }^{[41]}$.

\section{2 石墨烯表面 $\mathrm{sp}^{2}$ 碳的亲双烯体加成}

除活性自由基外，亲双烯体分子也可与石墨烯片层 上的 $\mathrm{sp}^{2}$ 碳发生加成反应, 例如: 亚甲胺叶立德可通过
1,3-环加成与石墨烯片层中的 $\mathrm{sp}^{2}$ 碳发生反应. 上述加成 反应已成功应用于系列纳米石墨烯基材料，甚至纳米碳 材料的功能化, 包括富勒烯、碳纳米管等. 目前通过亲 双烯体分子的加成反应，可制备得到多种共价功能化材 料, 在纳米复合材料、生物科技、纳米电子器件、药物 传递和太阳能电池等领域表现出极大的应用潜力. 2010 年, Georgakilas 等 ${ }^{[42]}$ 首先将制备所得石墨烯片层分散于 有机溶剂, 再通过叶立德的 1,3-环加成反应在石墨烯片 层表面引入吡咯环，最终通过与上述石墨烯片层上吡咯 环反应，得到二羟基苯基共价修饰的石墨烯片层，如图 2 所示. 石墨烯片层上引入大量的羟基，可有效改善其 在乙醇、 $N, N$-二甲基甲酰胺(DMF)等极性溶剂中的分散 性. Vadukumpully 等 ${ }^{[43]}$ 将烷基叠氮化合物作为亲双烯体 分子引入到石墨烯片层，从而得到烷基化合物共价修饰 石墨烯片层，其烷基化合物包括己基、十二烷基等，长 链烷基共价修饰的石墨烯复合材料在丙酮、甲苯等常见 有机溶剂中表现出较强的分散性. 此外, 石墨烯表面引 入含有羧基的烷基链，可有效固定金属纳米粒子，并将 金属纳米粒子作为标记来研究石墨烯片层表面反应位 点的分布. 此后也有许多科学家通过亲双烯体加成反应 制备得到了化学共价修饰的石墨烯衍生物, 有效地改善 其分散性等, 在多领域展现出多种多样的应用前 景 [44-48]. 石墨烯化学结构独特, 基本单元为六元环, 其组 成碳原子基本为饱和的 $\mathrm{sp}^{3}$ 碳, 直接对石墨烯表面的碳 原子进行共价修饰往往难以实现, 可应用的化学反应比

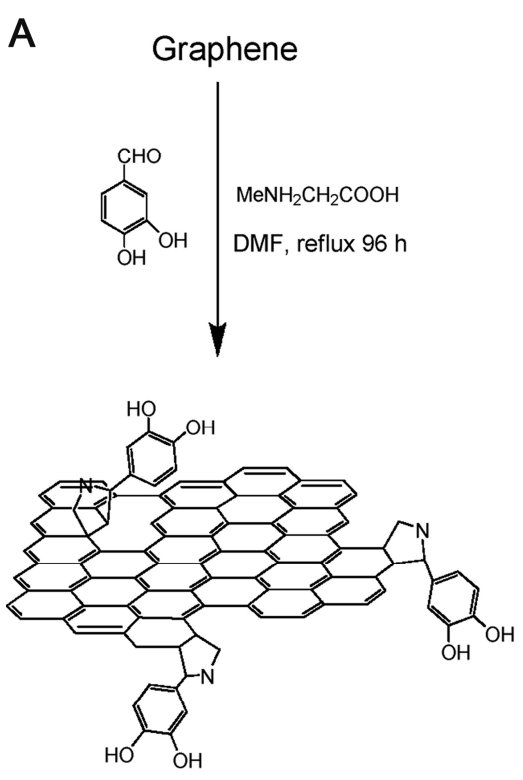

Graphene-f-OH

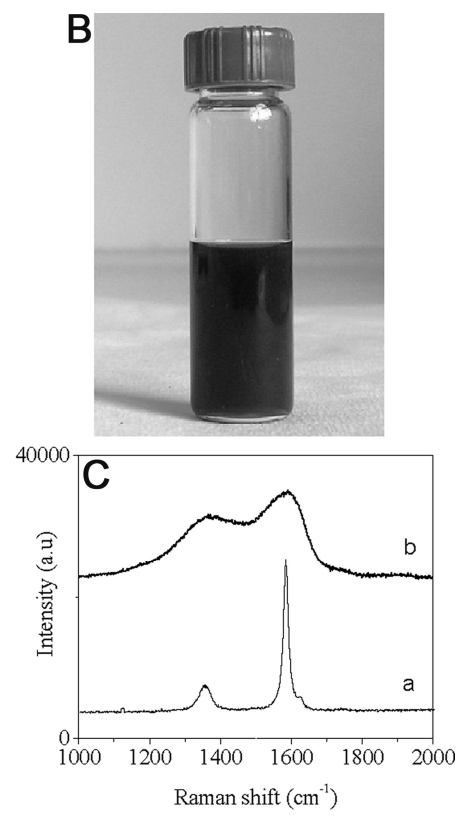

图 2 (A)石墨烯 1,3-环加成反应的示意图、(B)功能化石墨烯乙醇分散液和(C)石墨烯及功能石墨烯的拉曼光谱 ${ }^{[42]}$

Figure 2 (A) Schematic representation of 1,3-dipolar cycloaddition of azomethine ylide on grapheme, (B) dispersion of functionalized graphene in ethanol, and (C) Raman spectra of (a) graphene and (b) pyrrolidine functionalized graphene ${ }^{[42]}$ 
较受限. 通过对石墨烯表面缺陷处 $\mathrm{sp}^{2}$ 碳的直接加成有 助于实现石墨烯表面的共价修饰, 但是鉴于石墨烯表面 $\mathrm{sp}^{2}$ 碳数目较少, 反应活性位点有限, 直接对石墨烯表面 共价修饰的应用范围则更加有限. 采用先对其氧化衍生 物即氧化石墨烯, 先共价修饰再还原处理的方法, 可有 效地扩展共价修饰石墨烯类材料的化学途径, 得到多种 多样的化学共价修饰石墨烯类材料.

\section{2 氧化石墨烯的共价修饰}

氧化石墨烯是富含羟基、羧基、环氧基等含氧活性 基团的石墨烯的重要衍生物, 也是氧化还原法制备石墨 烯的重要前体. 氧化石墨烯表面富含活性含氧官能团, 由于 $\mathrm{sp}^{2}$ 碳与 $\mathrm{sp}^{3}$ 碳交叉分布造成表面存在大量缺陷, 这 为氧化石墨烯的化学共价修饰提供了足够的反应位点 及反应空间. 借助经典的有机反应, 将有机官能团与氧 化石墨烯富含的活性含氧基团反应形成新的共价键, 再 通过还原处理制备得到化学共价修饰的石墨烯复合材 料. 氧化石墨烯的共价修饰是借助氧化石墨烯表面和边 缘的活性含氧基团进行多种化学反应, 将有机小分子和 高分子聚合物借助共价键与氧化石墨烯片层连接, 通过 化学结构的改变实现对氧化石墨烯的共价修饰. 氧化石 墨烯结构中含有大量的羧基、羟基和环氧键等活性基团,
如图 3 所示，可以利用酯化反应、酰化反应、硅烷化反 应、Williamson 反应、Wittig 反应和 Claisen 重排反应等 多种经典有机反应对氧化石墨烯进行小分子和聚合物 的共价键功能化 ${ }^{[49]}$. 尤其在聚合物大分子方面，目前已 经实现了多类聚合物体系与石墨烯的成功复合，按照合 成策略可分为以下两类 ${ }^{[50]}$ : 向石墨烯接枝法 (graftingonto)和从石墨烯接枝法(grafting-from).

\section{1 酯化/酰化反应}

氧化石墨烯边缘的羧基提供了丰富的酰化、酯化的 反应位点，一般可通过与 $\mathrm{SOCl}_{2}$ 预处理，接着借助亲核 试剂进攻来实现, 或者借助碳二亚胺的偶联, 添加催化 量 4-二甲氨基吡啶(DMAP)或 $N$-差基琥珀酰亚胺(NHS) 等, 实现醇或胺类化合物对氧化石墨烯边缘羒基的共价 功能化. 氧化石墨烯边缘的羟基也可通过碳二亚胺的偶 联反应修饰，氧化石墨烯表面的羟基作为亲核试剂进攻 其他反应物的羧基等发生缩合反应. Liu 等 $^{[51]}$ 将氨基端 的寡聚噻吩通过酰胺化反应连接到氧化石墨烯片层，得 到了聚噻吩共价修饰的氧化石墨烯给受体材料, 与标准 器件 $\mathrm{C}_{60}$ 及聚噻吩相比，表现出更为优异的光电特性. $\mathrm{Yu}$ 等 ${ }^{[52]}$ 通过羟基端聚己基噻吩(P3HT)与氧化石墨烯边 缘的羧基发生酯化反应，制备得到 P3HT/氧化石墨烯复

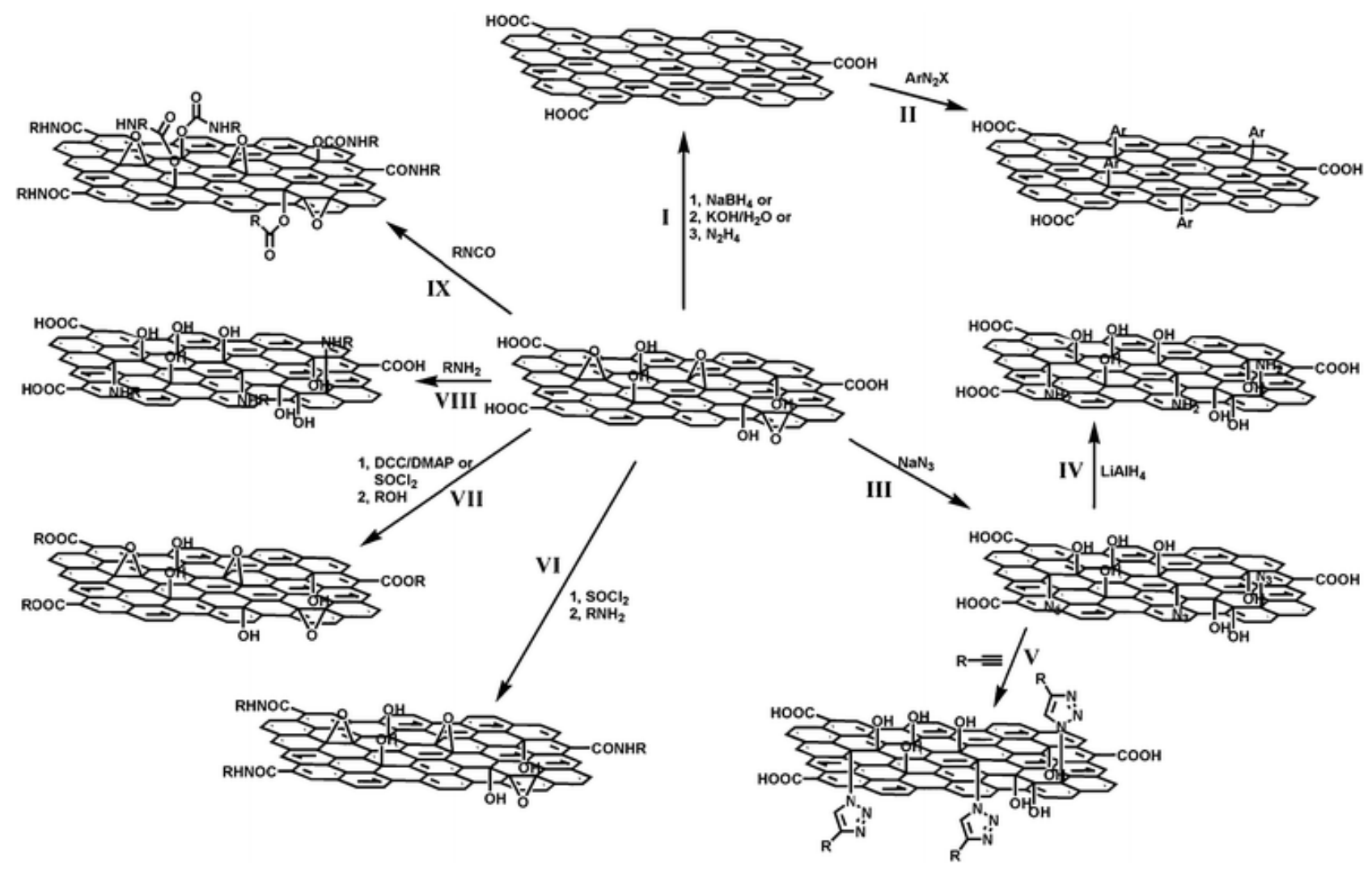

图 3 共价修饰氧化石墨烯的各类有机化学反应示意图 ${ }^{[49]}$

Figure 3 Schematic illustration showing various covalent functionalization chemistry of graphene oxide ${ }^{[49]}$ 
合材料, 如图 4 所示, 该复合材料可在多种常用有机溶 剂中有效分散. 此后, 该课题组将该复合材料用于光电 器件设计, 其光电效率相较于 P3HT 有着较大的提升.

\subsection{Williamson 反应}

氧化石墨烯的特殊化学结构使其具有独特的理化 性质. 氧化石墨烯含有多种活性含氧官能团, 主要包括 二维平面上的环氧基、羟基，平面边缘的羰基、醌基、 羧酸、酚基和酯基. 活性含氧基团的存在使得氧化石墨 烯具有较多的化学反应位点, 但是这些含氧基团的高化 学反应性也使得化学共价修饰过程难以控制, 同时可能 有多种反应同时进行. 2018 年, Isabella 等 ${ }^{[35]}$ 选择性地对 氧化石墨烯表面的羟基及羰基实现功能化. 如图 5 所示, 他们在较为温和的室温条件下, 通过 Williamson 反应实 现了氧化石墨烯表面羟基的醚化发应, 为氧化石墨烯的 多功能化开辟了新的思路, 该途径可用于设计多功能化 的石墨烯基纳米材料. 此后, 该团队又尝试对氧化石墨
烯表面的羰基通过 Wittig 反应实现功能化，但由于氧化 石墨烯表面的酮羰基较少，所以 Wittig 反应对氧化石墨 烯羰基的共价修饰较难实现. Isabella 等在温和条件下实 现对氧化石墨烯表面羟基的醚化, 为氧化石墨烯的化学 共价修饰提供了新的途径.

\subsection{Claisen 重排反应}

此外，可利用氧化石墨烯表面的活性羟基与 $N, N-$ 二 甲基乙酰胺二甲缩醛(DMDA)发生缩合反应. 通过上述 缩合反应, 如图 6 所示, 将氧化石墨烯表面的烯丙位羟 基原位转化为乙烯基醚中间体，接着通过 $[3,3] \sigma$ 重排的 方式转化形成新的碳一碳键 ${ }^{[53]}$. 通过氧化石墨烯表面的 Eschenmoser-Claisen [3,3] $\sigma$ 重排, 不仅在氧化石墨烯表 面形成新的 $\mathrm{C}-\mathrm{C}$ 键, 同时引入稳定的羰基, 这在功能 化石墨烯领域具有重要意义和实用性. 利用氧化石墨烯 表面的 Echenmoser-Claisen 重排，通过 DMDA 将其表面 羟基直接转化为可与平面 $\mathrm{sp}^{3}$ 碳连接的 $N, N$-二甲基酰胺

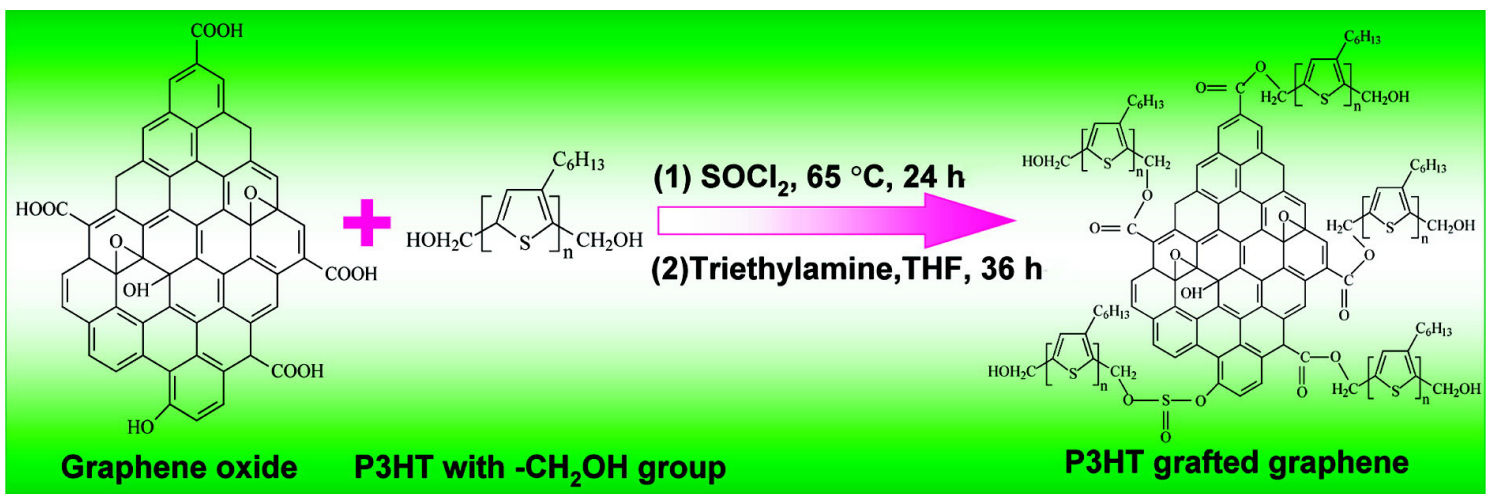

图 4 氧化石墨烯与羟基端的 P3HT 酯化化学反应示意图 ${ }^{[52]}$

Figure 4 Reaction between $\mathrm{GO}$ and $\mathrm{CH}_{2} \mathrm{OH}$-terminated $\mathrm{P} 3 \mathrm{HT}$ chains through an esterification reaction ${ }^{[52]}$

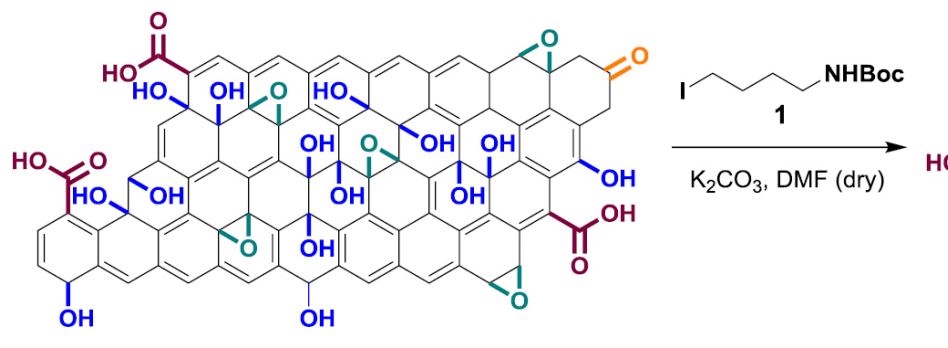

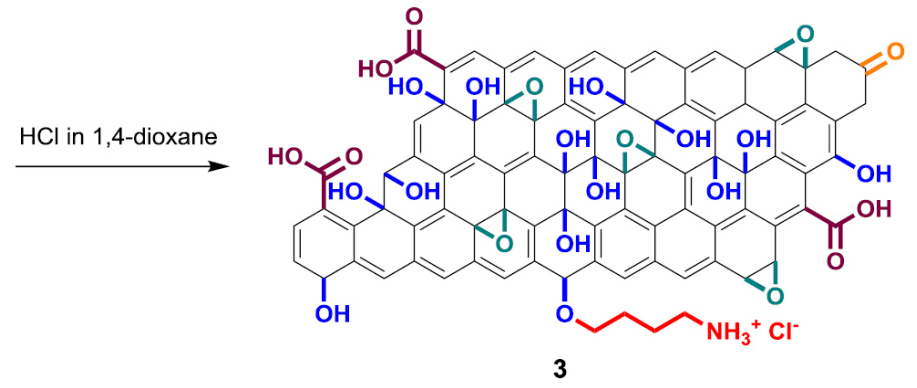

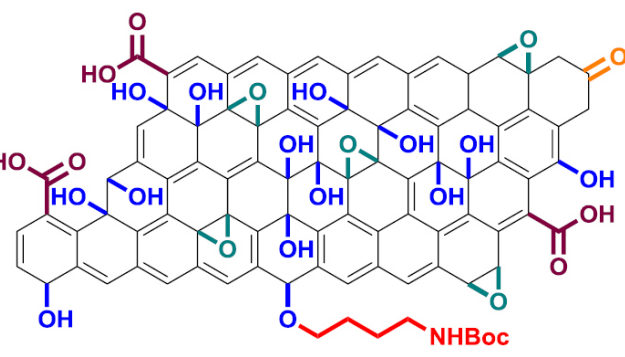

2

图 5 Williamson 反应共价修饰氧化石墨烯 ${ }^{[35]}$

Figure 5 Derivatization of GO by Williamson reaction ${ }^{[35]}$ 

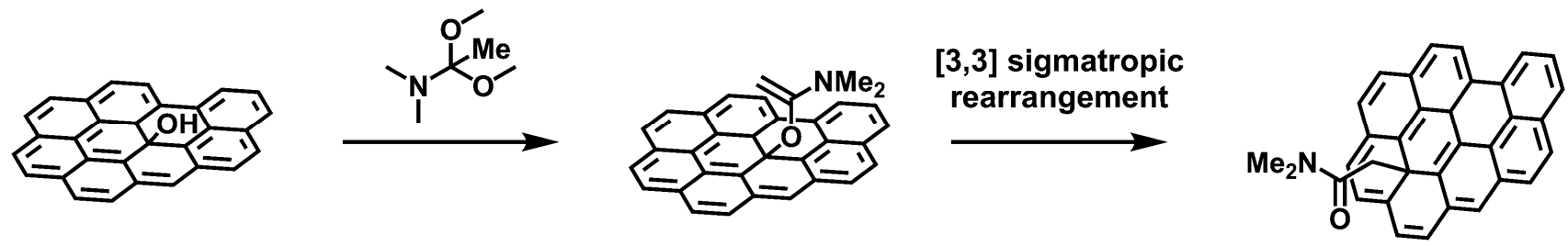

图 6 通过缩合以及 Eschenmoser-Claisen [3,3] $\sigma$ 重排修饰氧化石墨烯 ${ }^{[53]}$

Figure 6 Functionalization of GO via condensation followed by Eschenmoser-Claisen [3,3] $\sigma$ rearrangement ${ }^{[53]}$

基团，该类基团在强碱性条件下皇化，转化为相应的羧 酸盐, 可有效改善石墨烯衍生物在水中的分散性. 2019 年, David 小组 ${ }^{[54]}$ 采用相同策略, 通过共价修饰制备得 到甲基丙烯酸酯(MA)-氧化石墨烯纳米复合材料, 并成 功地将 MA 共价功能化的氧化石墨烯复合材料用于 3D 打印, 该复合材料可形成较为复杂的微观结构, 具有较 好的 3D 打印材料潜能, 并进一步扩展到其他领域.

\section{4 环氧开环反应}

氧化石墨烯表面的环氧基团也可作为化学共价修 饰的活性位点, 通过亲核试剂对环氧基团的开环反应, 可引入新的功能性基团, 增强其氧化石墨烯的功能性. 目前较为常用的亲核分子为胺类化合物，包括脂肪族、 芳香族和端基为氨基的聚合物分子. 该反应通常以氧化 石墨烯的极性溶剂分散液作为反应液在加热的条件下 进行. 如图 7 所示, $\mathrm{Niu}$ 等 ${ }^{[55}$ 将含有端氨基的离子液体通 过环氧开环反应成功引入到氧化石墨烯的表面, 得到了 氧化石墨烯片层衍生物, 该片层可在无任何聚合物或表 面活性剂的存在下, 稳定分散于 $\mathrm{H}_{2} \mathrm{O} 、 \mathrm{DMF}$ 和二甲基亚 砜(DMSO)等极性溶剂中. 其它亲核试剂也可与氧化石 墨烯表面的环氧基团发生开环反应. Swager 小组 ${ }^{[56]}$ 此前 报道过丙二腈的钠盐可与氧化石墨烯表面的环氧基团 反应, 与 $\mathrm{sp}^{2}$ 杂化碳形成新的 $\mathrm{C}-\mathrm{C}$ 键, 氧化石墨烯边缘
的羧酸具有一定的酸性，可以中和一部分丙二腈的钠盐 使其失活. 因此, 该反应需要使用过量丙二腈的钠盐 (约 5.5 equiv.)来保证环氧开环反应的发生.

\section{5 聚合物 “grafting-onto” 策略}

聚合物因其分子量大、分子链长、结构规整等特点, 在氧化石墨烯及石墨烯化学共价修饰制备复合高性能 材料方面占据了重要的地位. Wu 课题组 ${ }^{[57]}$ 此前采用向 石墨烯接枝法(grafting-onto)策略，通过氧化石墨烯上羧 基与嵌段共聚物 SEBS 发生酯化反应, 制备得到了 SEBS 聚合物功能化的氧化石墨烯, 该功能化的氧化石 墨烯可在四氢呋喃(THF)中与聚苯乙烯(PS)混合形成复 合材料. 该复合材料性能与未改性 PS 相比，拉伸强度 增加了 $78 \%$, 杨氏模量提高了 $73 \% .2008$ 年, Dai 小组 ${ }^{[58]}$ 利用酰卤的酯化反应，制备了具有生物相容性的聚乙二 醇(PEG)功能化的氧化石墨烯. PEG 的引入改善了氧化 石墨烯的水中分散性及生物相容性，该复合材料能够在 血浆等生理环境下保持稳定分散; 然后再利用 $\pi-\pi$ 相互 作用, 将抗癌药物喜树碱衍生物(SN38)负载到氧化石墨 烯上, 提高了抗肿瘤药物的生物利用度, 开启了氧化石 墨烯在生物医药方面的应用研究. 采用 grafting-onto 策 略得到的 PEG 共价修饰的氧化石墨烯, 在非水溶性抗 癌药物纳米运输领域发挥了极大的作用.

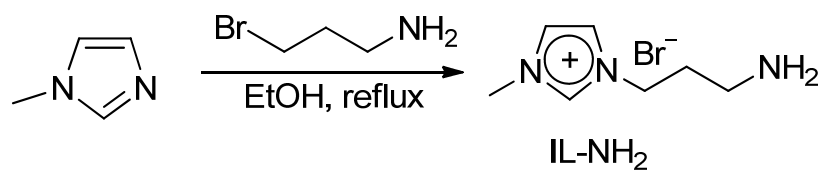

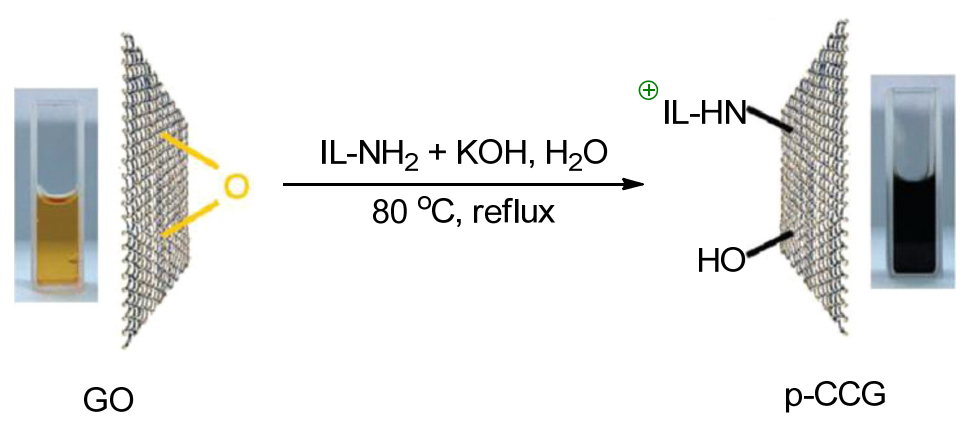

图 7 氨基端的离子液体在氧化石墨烯表面的开环反应 ${ }^{[55]}$

Figure 7 Ring-opening reactions between amino-ended ion liquids and graphene oxide ${ }^{[55]}$ 


\section{6 聚合物 “grafting-from” 策略}

聚合物共价修饰氧化石墨烯以及石墨烯表现出更 优越的热力学、导电、机械性能. 但与小分子共价修饰 相比, 由于活性基团含量低以及聚合物链的屏蔽作用等 限制聚合物链共价修饰的反应活性, 使得聚合物对氧化 石墨烯表面共价修饰的反应效率较低. 为避免聚合物共 价修饰接枝率低、反应效率低等问题, 科学家们采用从 石墨烯接枝法 (grafting-from), 也就是在石墨烯或其衍 生物上引入功能性分子, 引发单体聚合在片层本身得到 聚合物, 制备出大量氧化石墨烯/聚合物复合材料, 其中 应用最多的是原子转移自由基聚合(ATRP) ${ }^{[59-61]}$. 基于 ATRP 能够快速启动和在休眠种及活性自由基间达到动 态平衡的特点, ATRP 成为制备聚合物共价功能化氧化 石墨烯中较为实用的技术之一. Lee 等 ${ }^{[62]}$ 报道通过 2澳-2-甲基丙基溴(BMPB)的酰基溴与氧化石墨烯表面的 羟基反应, 制备了 ATRP 引发剂, 再引发苯乙烯、甲基 丙烯酸甲酯和丙烯酸甲酯等单体在氧化石墨烯表面的 聚合反应, 得到了聚合物功能化材料. 如图 8 所示, Liu 等 ${ }^{[63]}$ 采用 “grafting-from” 策略, 通过 SET-LRP 从石墨 烯表面原位引发 2-羟基丙烯酸差乙酯(HEA)单体聚合, 制备了聚合物 PHEA 共价功能化的石墨烯. 该工作首先 制备了表面含溴的石墨烯片层, 溴基的存在为随后的 SET-LRP 聚合提供了引发基团. SET-LRP 作为一种在低 温下高速进行, 并能有效控制大分子链生长的可控/ “活性” 自由基聚合技术, 可以相对高效且温和地实现聚 合物链在石墨烯材料上的可控共价功能化改性. 该聚合
物共价修饰的石墨烯复合材料在常规的有机溶剂中表 现出较好的分散性. 另外, PHEA 具有良好的水溶性、无 毒性以及生物相容性, 被广泛应用在生物载药领域, 该 复合材料也有望应用于生物载药领域. 除了 ATRP 和 SET-LRP 以外, 可逆加成一断裂链转移(RAFT) 聚合和 Ziegler-Natta 聚合也被应用于石墨烯的可控聚合物共价 修饰 ${ }^{[64]}$. 采用 “grafting-from” 策略可解决聚合物的大位 阻效应造成的低接枝率的问题, 但由于氧化石墨烯原位 聚合得到的高分子链结构不明确，没有办法对其结构进 行精确的表征.

\section{7 点击化学}

2001 年, Sharpless 等 ${ }^{[65-66]}$ 提出了点击化学 (click chemistry)这一化学合成的新思路, 利用廉价易得的原 料, 通过高效专一的化学反应实现分子单元之间的拼 接, 尤其强调开辟以碳一杂原子键 $(\mathrm{C}-\mathrm{X}-\mathrm{C})$ 合成为基 础的组合化学新方法. 此前, Hawker 等 ${ }^{[67-68]}$ 将这一策略 结合于材料科学, 制备得到性能优越的聚合物及复合材 料, 点击化学在高分子领域展现出作极大的优势. 因其 具有定量、高效、无副产物、无副反应、条件温和、应 用范围广等优点，被广泛应用于新型聚合物材料的设计 及功能性纳米材料的构建等方面 ${ }^{[69-70]}$. 此前有文献报 道, 尝试借助高效的点击化学, 在氧化石墨烯表面先进 行小分子偶联，引入聚合物，可高效地实现氧化石墨烯 的聚合物共价修饰，也有人尝试利用氮宾化学将系列叠 氮封端的聚合物偶联到氧化石墨烯的表面 ${ }^{[11-73]}$.

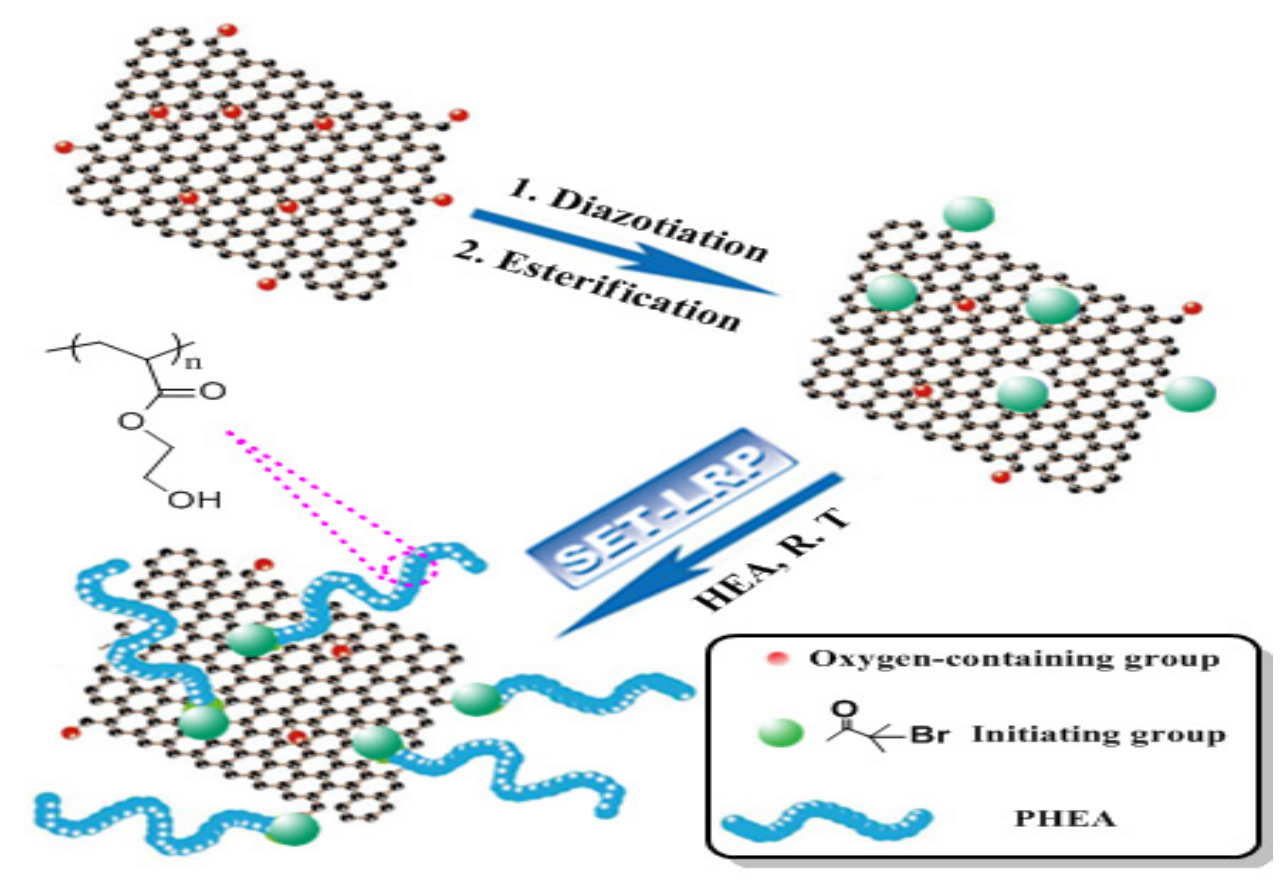

图 8 运用 “从上接枝” 策略通过 SET-LRP 原位制备 PHEA/graphene 复合材料 ${ }^{[63]}$

Figure 8 Preparation of PHEA/functionalized graphene via in situ SET-LRP with the grafting-from strategy ${ }^{[63]}$ 


\section{8 共价双官能化}

氧化石墨烯表面及边缘的活性含氧基团的化学共 价修饰有极大的应用前景, 共价修饰引入的聚合物和小 分子可调控氧化石墨烯的化学结构, 赋予氧化石墨烯新 的活性和功能. 利用有机反应, 人们设计和构造出许多 由石墨烯及氧化石墨烯衍生出的新型二维材料, 对综合 石墨烯及氧化石墨烯的优异性质、实现商业化应用具有 极其重要的意义. 除了单一功能化外, 共价双官能化还 可以通过不同的化学反应, 更好地控制功能分子在氧化 石墨烯表面的特定修饰. 2018 年, Imani 等 ${ }^{[74]}$ 借助氧化石 墨烯边缘羧基的酰胺化反应, 制得了精氨酸和 PEG 双 功能化氧化石墨烯, 但是反应条件苛刻, 过程难以控制. Ménard-Moyon 等 ${ }^{[75]}$ 提出了一种温和碱性条件下氧化石 墨烯的双官能化方法, 如图 9 所示, 该方法通过氧化石 墨烯表面环氧基团和羟基的连续可控的共价化学修饰, 实现温和条件下氧化石墨烯的双官能化. 该方法简单易 行, 反应条件温和, 使得氧化石墨烯的独特结构和性能 得以保存，同时在氧化石墨烯表面引入多个活性基团， 增强了氧化石墨烯的功能性. 该策略可用于制备多功能 化氧化石墨烯, 在材料科学、生物医学、能源储备等许 多领域具有潜在的应用前景.

石墨烯是二维单片层碳材料, 具有独特的化学结 构, 表现出优异的电学、力学和热学等理化性质, 使得 石墨烯很快就成为在吸附材料、传感器、能源等领域最 具应用前景的材料之一. 此外, 氧化石墨烯具有类似石 墨烯的单片层结构, 同时富含羟基、羧基、环氧基等含
氧官能团. 利用活性含氧基团、借助有机小分子、生物 大分子、功能性纳米颗粒或聚合物, 通过共价化学实现 氧化石墨烯的功能化，一方面，提高氧化石墨烯的理化 性能，扩展氧化石墨烯在生物、能源等领域的应用; 另 一方面，共价修饰的氧化石墨烯通过还原可得到共价修 饰的石墨烯材料, 实现对石墨烯表面的功能化，相较于 直接对石墨烯片层的化学共价修饰，反应条件温和，选 择性多，适用范围广，可有效地实现石墨烯类材料应用 的多样性

\section{3 结论与展望}

综上所述，目前发展了一系列具有代表性的有机化 学方法, 包括酯化反应、酰化反应、Williamson 反应和 Claisen 重排反应、ATRP、点击化学等, 将有机小分子 和聚合物分子链借助共价键引入到石墨烯及氧化石墨 烯片层, 通过化学结构的改变实现对石墨烯及氧化石墨 烯的修饰，提升石墨烯类复合材料的功能性. 与非共价 相互作用制备得到石墨烯基复合材料相比，如 $\pi-\pi$ 作 用、疏水相互作用和氢键相互作用等，通过化学反应实 现石墨烯及氧化石墨烯的共价功能化，一方面结构稳 定、反应高效、条件可控，一方面可增加其表面活性反 应位点，增强其功能性，扩展石墨烯及氧化石墨烯在多 个领域的应用. 化学共价修饰的石墨烯类材料结构稳 定、反应可控、性能优越，制备所得复合材料兼容多种 优异性能，在信息、能源、生物等领域有着极大的应用 潜能. 如何在温和的条件下保留石墨烯原有的独特理化
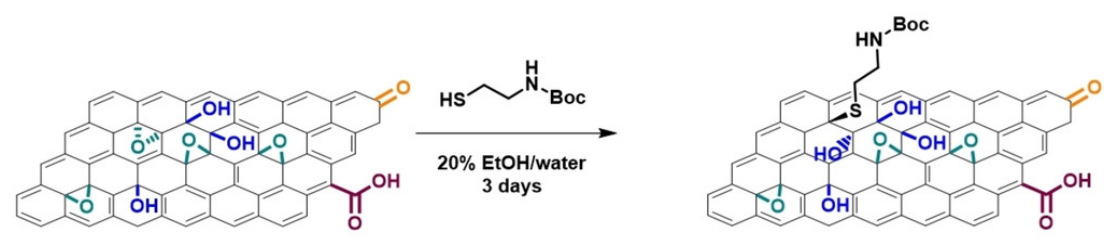

1
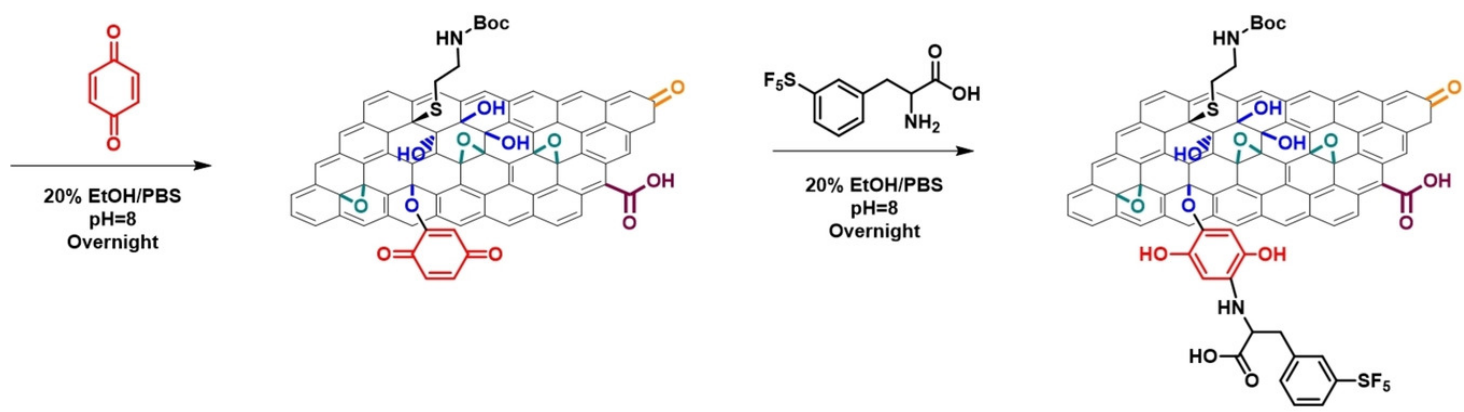

3

图 9 化学共价修饰双官能化氧化石墨烯示意图 ${ }^{[75]}$

Figure 9 Double covalent functionalization of GO via organic reactions ${ }^{\text {[75] }}$ 
性质, 多步反应可控进行, 实现石墨烯表面的绿色多样 官能化, 这是石墨烯及氧化石墨烯的化学共价修饰所面 临的挑战, 也将为共价修饰石墨烯及氧化石墨烯类复合 材料带来新的机遇.

\section{References}

[1] Peierls, R. E. Ann. Inst. Henri Poincare, Sect. A 1935, 5, 177.

[2] Mermin, N. D. Phys. Rev. 1968, 176, 250.

[3] Novoselov, K. S.; Geim, A. K.; Morozov, S. V.; Grigorieva, I. V.; Firsov, A. Science 2004, 306, 666

[4] Geim, A. K.; Novoselov, K. S. Nat. Mater. 2007, 6, 183.

[5] Novoselov, K. S.; Falko, V. I.; Colombo, L.; Gellert, P. R.; Schwab, M. G.; Kim, K. Nature 2012, 490, 192.

[6] Geim, A. K. Science 2009, 324, 1530.

[7] Zhu, Y. W.; Murali, S.; Cai, W. W.; Li, X. S.; Suk, J. W.; Potts, J. R.; Ruoff, R. S. Adv. Mater. 2010, 22, 3906.

[8] Lee, C.; Wei, X.; Kysar, J. W.; Hone, J. Science 2008, 321, 385.

[9] Niimi, Y.; Matsui, T.; Kambara, H.; Tagami, K.; Tsukadaand, M.; Fukuyama, H. Phys. Rev. B: Condens. Matter Mater. Phys. 2006, 73, 085421.

[10] Balandin, A. A.; Ghosh, S.; Bao, W. Z.; Calizo, I.; Teweldebrhan, D.; Miao, F.; Lau, C. N. Nano Lett. 2008, 8, 902.

[11] Berger, C.; Song, Z. M.; Li, T. B.; Li, X. B.; Ogbazghi, A. Y.; Feng, R.; Dai, Z. T.; Marchenkov, A. N.; Conrad, E H.; First, P. N.; de Heer, W. A. J. Phys. Chem. B 2004, 108, 19912.

[12] Schedin, F.; Geim, A. K.; Morozov, S. V.; Hill, E. W.; Blake, P.; Katsnelson, M. I.; Novoselov, K. S. Nat. Mater. 2007, 6, 652.

[13] Li, Z. Y.; Zhang, W. H.; Luo, Y.; Yang, J. L.; Hou, J. G. J. Am. Chem. Soc. 2009, 131, 6320 .

[14] Stankovich, S.; Piner, R. D.; Nguyen, S. T.; Ruoff, R. S. Carbon 2006, 44, 3342.

[15] Titelman, G. I.; Gelman, V.; Bron, S.; Khalfin, R. L.; Cohen, Y.; Bianco-Peled, H. Carbon 2005, 43, 641.

[16] Szabo, T.; Tombacz, E.; Illes, E.; Dekany, I. Carbon 2006, 44, 537.

[17] He, H., Riedl, T., Lerf, A.; Klinowski, J. J. Phys. Chem. 1996, 100 , 19954.

[18] Misra, S. K.; Kondaiah, P.; Bhattacharya, S.; Rao, C. N. R. Small 2012, 8,131.

[19] Song, Y.; Wei, W.; Qu, X. Adv. Mater. 2011, 23, 4215.

[20] Li, J. L.; Bao, H. C.; Hou, X. L.; Sun, X.; Wang, G.; Gu, M. Angew. Chem., Int. Ed. 2012, 51, 1830.

[21] Shao, Y.; Wang, J.; Wu, H.; Liu, J.; Aksay, I. A.; Lin, Y. Electroanalysis 2010, 22, 1027 .

[22] Szabó, T.; Berkesi, O.; Forgó, P.; Josepovits, K.; Sanakis, Y.; Petridis, D.; Dékány, I. Chem. Mater. 2006, 18, 2740.

[23] Lerf, A.; He, H.; Riedl, T.; Forster, M.; Klinowski, J. Solid State Ionics 1997, 101 103, 857.

[24] Dua, V.; Surwade, S. P.; Ammu, S.; Agnihotra, S. R.; Jain, S.; Roberts, K. E.; Park, S.; Ruoff, R. S.; Manohar, S. K. Angew. Chem., Int. Ed. 2010, 49, 2154.

[25] David, L.; Bhandavat, R.; Singh, G. ACS Nano 2014, 8, 1759.

[26] Shamsipur, M.; Molaei, K.; Molaabasi, F.; Hosseinkhani, S.; Taherpour, A.; Sarparast, M.; Moosavifard, S. E.; Barati, A. ACS Appl. Mater. Interfaces 2019, 11, 46077.

[27] Cao, Y.; Lai, Z.; Feng, J.; Wu, P. J. Mater. Chem. 2011, 21, 9271.

[28] Xu, Z. Y.; Li, Y. J.; Shi, P.; Wang, B. J.; Huang, X. Y. Chin. J. Org. Chem. 2013, 33, 573 (in Chinese). (徐志远, 李永军, 史萍, 王博婵, 黄晓宇, 有机化学, 2013, 33, 573.)

[29] Dai, J.; Lang, M. D. Acta Chem. Sinica 2012, 70, 1237 (in Chinese). (戴静, 郎美东, 化学学报, 2012, 70, 1237.)

[30] Xu, Z. Y.; Wang, S.; Li, Y. J.; Wang, M. W.; Shi, P.; Huang, X. Y. ACS Appl. Mater. Interfaces 2014, 6, 17268.

[31] Stankovich, S.; Piner, R. D.; Chen, X.; Wu, N.; Ruoff, R. S. J.
Mater. Chem. 2006,16, 155.

[32] Bai, H.; Li, C.; Wang, X.; Shi, G. Chem. Commun. 2010, 46, 2376.

[33] Yoon, S.; In, I. J. Mater. Sci. 2011, 46, 1316.

[34] Cano, M.; Khan, U.; Sainsbury, T.; Neill, A. O.; Wang, Z.; McGovern, I. T.; Maser, W. K.; Benito, A. M.; Coleman, J. N. Carbon 2013, 52, 363.

[35] Vacchi, I. A.; Raya, J.; Bianco, A.; Ménard-Moyon, C. 2D Mater. 2018, 5, 035037.

[36] Ji, P.; Zhang, W.; Ai, S.; Zhang, Y.; Liu, J.; Liu, J.; He, P.; Li, Y. Nanotechnology 2019, 30, 115701.

[37] Sydlik, S. A.; Swager, T. M. Adv. Funct. Mater. 2013, 23, 1873.

[38] Vacchi, I. A.; Spinato, C.; Raya, J.; Bianco, A.; Ménard-Moyon, C. Nanoscale 2016, 8, 13714.

[39] Sinitskii, A.; Dimiev, A.; Corley, D. A.; Fursina, A. A.; Kosynkin, D. V.; Tour, J. M. ACS Nano 2010, 4, 1949.

[40] Hamilton, C. E.; Lomeda, J. R.; Sun, Z. Z.; Tour, J. M.; Barron, A. R. Nano Lett. 2009, 9, 3460.

[41] Deng, Y.; Li, Y. J.; Dai, J.; Lang, M. D.; Huang, X. Y. J. Polym. Sci. Part A: Polym. Chem. 2011, 49, 4747.

[42] Georgakilas, V.; Bourlinos, A. B.; Zboril, R.; Steriotis, T. A.; Dallas, P.; Stubos, A. K.; Trapalis, C. Chem. Commun. 2010, 46, 1766.

[43] Vadukumpully, S.; Gupta, J.; Zhang, Y.; Xu, C. Q.; Valiyaveettil, S. Nanoscale 2011, 3, 303.

[44] Nemes-Incze, P.; Osváth, Z.; Kamarás, K.; Biro, L. P. Carbon 2008, $46,1435$.

[45] Zhang, X.; Hou, L.; Cnossen, A.; Coleman, A. C.; Ivashenko, O.; Rudolf, P.; van Wees, B. J.; Browne, W. R.; Feringa, B. L. Chem.-Eur. J. 2011, 17, 8957.

[46] Quintana, M.; Spyrou, K.; Grzelczak, M.; Browne, W. R.; Rudolf, P.; Prato, M. ACS Nano 2010, 4, 3527.

[47] Liu, L. H.; Lerner, M. M.; Yan, M. Nano Lett. 2010, 10, 3754.

[48] Zhong, X.; Jin, J.; Li, S.; Niu, Z.; Hu, W.; Li, R.; Ma, J. Chem. Commun. 2010, 46, 7340.

[49] Loh, K. P.; Bao, Q.; Anga, P. K. Yang, J. X. J. Mater. Chem. 2010, 20, 2277.

[50] Salavagione, H. J.; Martínez, G.; Ellis, G. Macromol. Rapid. Commun. 2011, 32, 1771.

[51] Liu, Y.; Zhou, J.; Zhang, X.; Liu, Z.; Wan, X.; Tian, J.; Wang, T.; Chen, Y. Carbon 2009, 47, 3113.

[52] Yu, D.; Yang, Y.; Durstock, M.; Baek, J. B.; Dai, L. ACS Nano 2010, 4, 5633 .

[53] Collins, W. R.; Lewandowski, W.; Schmois, E.; Walish, J.; Swager, T. M. Angew. Chem., Int. Ed. 2011, 50, 8848

[54] Palaganas, J. O.; Palaganas, N. B.; Ramos, L. J. I.; David, C. P. C. ACS Appl. Mater. Interfaces 2019, 11, 46034.

[55] Yang, H.; Shan, C.; Li, F.; Han, D.; Zhang, Q.; Niu, L. Chem. Commun. 2009, 26, 3880.

[56] Collins, W. R.; Schmois, E.; Swager, T. M. Chem. Commun. 2011, 47, 8790 .

[57] Cao, Y.; Lai, Z.; Feng, J.; Wu, P. J. Mater. Chem. 2011, 21, 9271.

[58] Liu, Z.; Robinson, J. T.; Sun, X.; Dai, H. J. Am. Chem. Soc. 2008, 130, 10876.

[59] Lee, S. H.; Kim, H. W.; Hwang, J. O.; Lee, W. J.; Kwon, J.; Bielawski, C. W.; Ruoff, R. S.; Kim, S. O. Angew. Chem., Int. Ed. 2010, 49, 10084

[60] Wang, D.; Ye, G.; Wang, X.; Wang, X. Adv. Mater. 2011, 23, 1122.

[61] Fang, M.; Wang, K.; Lu, H. B.; Yang, Y. L.; Nutt, S. J. Mater. Chem. 2009, 19, 7098.

[62] Lee, S. H.; Dreyer, D. R.; An, J.; Velamakanni, A.; Piner, R. D.; Park, S.; Zhu, Y.; Kim, S. O.; Bielawski, C. W.; Ruoff, R. S. Macromol. Rapid. Commun. 2010, 31, 281.

[63] Liu, Z. Z.; Zhu, S. J.; Li, Y. J.; Li, Y. S.; Shi, P.; Huang, Z.; Huang, X. Y. Polym. Chem. 2015, 6, 311 .

[64] Huang, Y.; Qin, Y.; Zhou, Y.; Niu, H.; Yu, Z. Z.; Dong, J. Y. Chem Mater. 2010, 22, 4096

[65] Kolb, H. C.; Finn, M. G.; Sharpless, K. B. Angew. Chem., Int. Ed. 2001, 44, 2004.

[66] Becer, C. R.; Hoogenboom, R.; Schubert, U. S. Angew. Chem., Int. 
Ed. 2009, 48, 4900.

[67] Wu, P.; Feldman, A. K.; Nugent, A. K.; Hawker, C. J.; Scheel, A.; Voit, B.; Pyun, J.; Frechet, J. M.; Sharpless, K. B.; Fokin, V. V. Angew. Chem., Int. Ed. 2004, 43, 3928.

[68] Helms. B.; Mynar, J. L.; Hawker, C. J.; Frechet, J. M. J. Am. Chem. Soc. 2004, 126, 15020.

[69] John, E.; Moses, A.; Moorhouse, D. Chem. Rev. 2007, 36, 1249.

[70] Zhang, T.; Zheng, C. H.; Ding, X. B.; Peng, Y. X. Prog. Chem. 2008, 20, 1090 (in Chinese)

(张涛, 郑朝辉, 成煦, 丁小斌, 彭宇行, 化学进展, 2008, 20,
1090.)

[71] He, H.; Gao, C. Chem. Mater. 2010, 22, 5054.

[72] Shen, J.; Hu, Y.; Li, C.; Qin, C.; Ye, M. S Small 2009, 5, 82.

[73] Pan, Y.; Bao, H.; Sahoo, N. G.; Wu, T.; Li, L. Adv. Funct. Mater. 2011, 21, 2754.

[74] Imani, R.; Prakash, S.; Vali, H.; Faghihi, S. Biomater. Sci. 2018, 6, 1636.

[75] Guo, S.; Nishina, Y.; Bianco, A.; Cécilia, M.-M. Angew. Chem., Int. Ed. 2020, 59, 1542 . 